Physiological Aspects of Legged Terrestrial Locomotion 
Giovanni Cavagna

\section{Physiological Aspects of Legged Terrestrial Locomotion}

The Motor and the Machine

照 Springer 


\author{
Giovanni Cavagna \\ Department of Physiology \\ Milano State University \\ Milan \\ Italy
}

ISBN 978-3-319-49979-6

ISBN 978-3-319-49980-2 (eBook)

DOI 10.1007/978-3-319-49980-2

Library of Congress Control Number: 2017930161

(C) Springer International Publishing AG 2017

This work is subject to copyright. All rights are reserved by the Publisher, whether the whole or part of the material is concerned, specifically the rights of translation, reprinting, reuse of illustrations, recitation, broadcasting, reproduction on microfilms or in any other physical way, and transmission or information storage and retrieval, electronic adaptation, computer software, or by similar or dissimilar methodology now known or hereafter developed.

The use of general descriptive names, registered names, trademarks, service marks, etc. in this publication does not imply, even in the absence of a specific statement, that such names are exempt from the relevant protective laws and regulations and therefore free for general use.

The publisher, the authors and the editors are safe to assume that the advice and information in this book are believed to be true and accurate at the date of publication. Neither the publisher nor the authors or the editors give a warranty, express or implied, with respect to the material contained herein or for any errors or omissions that may have been made. The publisher remains neutral with regard to jurisdictional claims in published maps and institutional affiliations.

Printed on acid-free paper

This Springer imprint is published by Springer Nature

The registered company is Springer International Publishing AG

The registered company address is: Gewerbestrasse 11, 6330 Cham, Switzerland 
To My Students 


\section{Foreword}

"Physiological Aspects of Legged Terrestrial Locomotion: The Motor and the Machine" is part textbook, part laboratory manual, and part biography. Cavagna takes the reader on his personal journey of discovery through muscular time and space. Time starting in the late 1960s with experiments performed on muscle heat production, and using Levin-Wyman ergometers (an instrument using floor-to-ceiling springs, pneumatic cylinders and mechanical catches) to control tiny levers imparting length changes on isolated frog muscles, all the way up to today. Space encompassing all the levels of muscle integration from proteins (myosin heads) to whole animals.

The first part of the book concerns muscle in vitro. Cavagna performed all of his muscle experiments in his laboratory at the Istituto di Fisiologia Umana at the Università di Milano. He built his own experimental apparatuses, starting with the aforementioned Levin-Wyman ergometer making measurements on whole frog muscles, and culminating in a home-made voice-coil lever system capable of performing controlled length changes of up to $0.2 \mathrm{~mm}$ in $100 \mu \mathrm{s}$. This lever system was for making measurements on a small segment of an isolated frog muscle fibre (i.e. an individual muscle cell) which was delimited by two laser-illuminated 'windows' $\sim 1 \mathrm{~mm}$ apart on the fibre. An optical and electronic system was capable of counting, up or down, the number of striations that passed by each window during a contraction. If the count was the same in the two windows, then the fibre segment between the two windows was isometric; if the count was greater in one window then the segment was lengthening, or if the count was less then the segment was shortening. A custom computer controlled the lever system in either length or force feedback to deliver precise isometric or isotonic conditions to the fibre segment. The results of these experiments are explained in Chap. 4.

The second part of the book concerns muscles in vivo. Cavagna pioneered the use of force platforms for the measurement of ground reaction forces during one or more complete locomotory cycles (step, stride, hop). In the mid-1970s Cavagna went to C. Richard (Dick) Taylor's Concord Field Station at Harvard to pass the technique on and to do external work measurements on small animals. Taylor returned the favour, going to Milano to measure the external work on medium-sized 
animals. More recently Cavagna went to Thailand to measure the external work on very large animals, Asian elephants, using a $2 \mathrm{~m}$ by $8 \mathrm{~m}$ platform. The database built up by these force platform experiments have continued to serve Cavagna right up to this day (Chaps. 6-8 and 10).

Only Cavagna has the breadth of personal experience, accumulated over many decades of hands-on experimentation involving proteins to pachyderms, to put everything together into one conceptual scheme. This book will appeal to advanced undergraduates, graduate students and professionals alike. The references at the end of each chapter not only illustrate Cavagna's contribution but also provide key papers for starting further in-depth research.

Brussels, Belgium

Norman Heglund

December 2016 


\section{Preface}

Looking backward over more than fifty years of experimental work on muscle and locomotion made on the same line of thought, I felt the need to join in a logical sequence otherwise scattered results whose common link was lost. I thought that if this was what I needed, it could also be useful for others interested in the field. This connection is made in this book where Physiological Aspects of Legged Terrestrial Locomotion are treated in the view of the two ingredients involved in locomotion: The Motor and the Machine, i.e. the muscle and the lever system of the limbs.

Muscular function has been classically described mainly by its capacity to convert the energy set free by chemical reactions into positive mechanical work and heat, i.e. its motor function. Furthermore, muscular function is usually treated separately from the function of the locomotor system. I hope that this book will show the limits of this approach, how the braking function of muscle is fundamental as its motor function during exercise and how the knowledge of both these functions, and particularly of their interaction, naturally blends with the study of the motion of the body as a whole.

In the two parts of this book, "Muscle: The Motor" and "Locomotion: MotorMachine Interaction", I attempted to put evidence on the one side the relationship between the fundamental properties of muscle and their manifestation in muscular exercise and, on the other side, the role that the mechanics of the exercise has in modifying the mechanism of muscle operation: This last effect resulted in the subdivision of the exposition of muscle physiology in two distinct sections of the first part of this book. In both sections, muscle physiology is treated almost exclusively from a mechanical point of view: The chemical processes that fuel muscular contraction are considered solely in their thermodynamic balance, not in their identification and kinetics.

The second part of this book shows how the operation of the machine is affected during locomotion by the limits set by the functional characteristics of muscle. The two basic mechanisms used to minimize energy expenditure, the pendular mechanism of walking and the bouncing mechanism of running, trotting and hopping, are treated in two different sections. In both cases locomotion is described in different experimental conditions: Speed of progression, step frequency, age, body 
mass and gravity by measuring the motion of the body as a whole, without a description of the movement of the individual body segments that cause it.

I hope that this book may help physiology, biology and physical education teachers and graduate students to get a synthetic, but comprehensive description of the mechanics of muscle contraction and of legged terrestrial locomotion. The several questions left unanswered may provide a stimulus to researchers for further experimental and analytical work. For this reason, particular attention is given to the description of the methods used in the experiments on isolated muscle specimens and on the whole body. Elementary knowledge of mathematics and physics is sufficient to understand this book.

I wish to thank Andrea Cavagna for several useful suggestions and Mario Legramandi for his careful revision of the final draft of this book.

Milan, Italy

Giovanni Cavagna 


\section{Contents}

\section{Part I Muscle: The Motor}

1 Experimental Procedures in the Study of Muscle Mechanics. . . . . . 9

$1.1 \quad$ Muscle Chamber and Stimulation . . . . . . . . . . . . . . . . 9

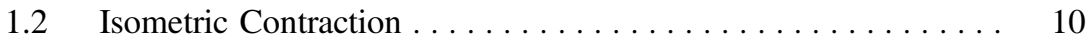

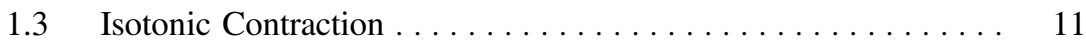

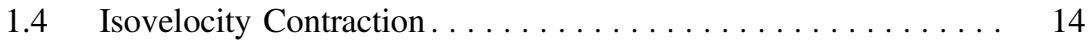

1.5 Single Muscle Fiber and Fiber Segment. . . . . . . . . . . . . . . . . . . . . . . . 15

1.6 Response of a System to an Action .............. 18

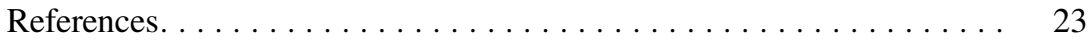

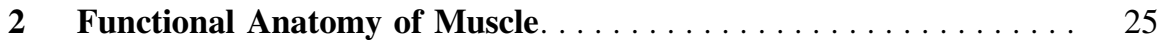

$2.1 \quad$ Structures in Series and in Parallel ............... 25

2.2 Localization of the "Motor" and of the Undamped Elastic Elements . . . . . . . . . . . . . . . . . . . . . . . . . . 29

2.3 Elastic Elements Having the Function of Containing and Centering the Contractile Component ........... 32

References............................. 34

3 Measurements Made During or Starting from a State

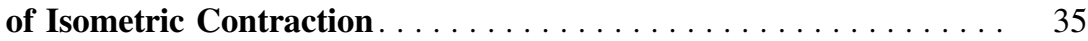

3.1 Phases of Muscular Contraction Determined on the Whole Muscle .......................... 35

3.2 Stress-Strain Diagram of the Apparent Elastic Elements Determined on the Whole Muscle . . . . . . . . . . . . . 36

3.3 Twitch, Clonus and Tetanus . . . . . . . . . . . . . . 39

3.4 Force-Length Relation (Isometric Contraction) . . . . . . . . . . 43

3.5 Functional Consequences of the Force-Length Relation . . . . . . 46

3.5.1 Equilibrium Conditions . . . . . . . . . . . . . 46

3.5.2 Limitation of the Movement Created

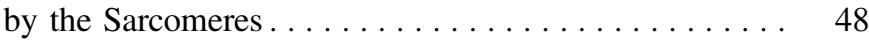


3.6 Force-Velocity Relation (Isotonic and Isovelocity

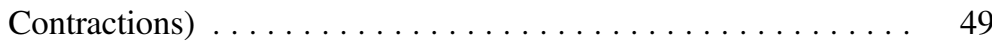

3.6.1 Experimental Procedure. . . . . . . . . . . . . . . 49

3.6.2 Description of the Force-Velocity Diagram . . . . . . . 50

3.6.3 Effect of Muscle Length . . . . . . . . . . . . 51

3.6.4 Force-Velocity of Shortening Relation at Different Times Since the Beginning of Stimulation ........ 52

3.6.5 General Meaning of the Force-Velocity of Shortening Relation....................... 52

3.6.6 Theoretical Interpretation of the Force-Velocity

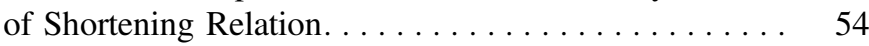

3.7 Functional Consequences of the Force-Velocity Relation . . . . . 58

$3.7 .1 \quad$ Power . . . . . . . . . . . . . . . . . . . . . 58

3.7.2 Cost of Positive and Negative Work . . . . . . . . . 59

3.8 Dynamic Force-Length Diagram (Iso-velocity Contraction) ... . 59

3.9 Phases of Muscular Contraction Determined on the Single

Muscle Fiber. . . . . . . . . . . . . . . . . . . . . . 61

3.9.1 Force-Length Diagram of the Undamped Structure Within the Sarcomere .................... 63

3.9.2 Force-Length Diagram of the Damped Structure Within the Sarcomere .................. 65

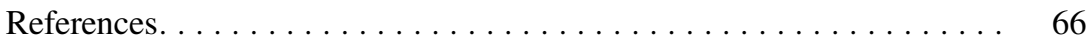

4 Measurements Made After Stretching the Contracting Muscle . . . . 69

4.1 Evidence of an Enhancement of Positive Work Production

by a Previously Stretched Muscle. . . . . . . . . . . . . . . . 69

4.2 What is the Origin of the Extra Work Done by a Previously Stretched Muscle?. . . . . . . . . . . . . . . . . . . . . . . . . 72

4.3 Experiments Made on the Whole Muscle............. 73

4.3.1 Mechanical Work and Efficiency in Isolated Frog and Rat Muscle................... 73

4.3.2 The Apparent Enhancement of the Contractile Component ..................... 74

4.3.3 Modification of the Apparent Elastic Characteristics

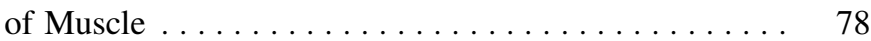

4.3.4 Physiological Meaning of the Modification of the Apparent Elastic Characteristics of Muscle . . . . .

4.3.5 Effect of Temperature on the Kinetics of the Fall in Force After Stretching (Stress-Relaxation). . . . . . . .

4.3.6 Effect of a Time Interval Between Stretching

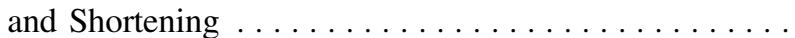


4.4 Experiments Made on the Single Muscular Fiber . . . . . . . . . . 86

4.4.1 Effect of Temperature and of the Velocity of Lengthening on the Kinetics of the Fall in Force After Stretching. . . . . . . . . . . . . . . . . 86

4.4.2 The Four Phases of Shortening Against the Maximal Isometric Force Taking Place After a Ramp Stretch . . 88

4.4.3 Effect of a Time Interval Between End of Stretching and Release to the Maximal Isometric Force. . . . . . . . 90

4.5 Experiments Made on a Tendon-Free Segment of the Muscular Fiber................................. 9

4.5.1 Transient Shortening Against the Maximal Isometric Force Is not Due to Stress-Relaxation of Tendons. . . . 91

4.5.2 Transient Shortening Against the Maximal Isometric Force Is Independent of the Velocity of Stretching . . . .

4.5.3 Transient Shortening Against the Maximal Isometric Force Is Independent of Sarcomere Stiffness . . . . . . . .

4.5.4 Transient Shortening Against the Maximal Isometric Force also Occurs When the Ramp Stretch Takes Place on the Ascending Limb of the Force-Length Relation. . .

4.5.5 Energy Transfer During Stress Relaxation Following Sarcomere Stretch . . . . . . . . . . . . . . . 98

4.6 Interpretation of the Experimental Results: Conclusive Remarks . . . . . . . . . . . . . . . . . . . . . . . . . . 103

4.7 Differences Between In Vitro and In Vivo Conditions . . . . . . . 105

4.7.1 Characteristics of the Movement Imposed to the Muscle. . . . . . . . . . . . . . . . . . . . . . . . 106

4.7.2 Effect of a Sub Maximal Stimulation. . . . . . . . . . 106

References. . . . . . . . . . . . . . . . . . . . . . . 107

5 Muscle Thermodynamics . . . . . . . . . . . . . . . . . . . . . . . . . . . 109

5.1 Interpretation of the Heat Exchanges Between Muscle

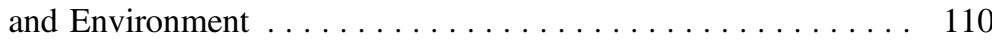

5.2 Methods of Heat Measurement. . . . . . . . . . . . . . . 114

5.3 Resting Heat . . . . . . . . . . . . . . . . . . . . . 115

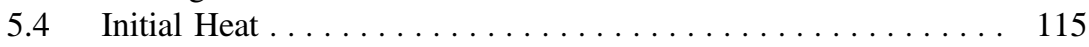

5.4.1 Activation and Maintenance Heat . . . . . . . . . 115

5.4 .2 Shortening Heat. . . . . . . . . . . . . . . . . . 117

5.4.3 Fenn Effect: A Connection Between Heat Production and the Force-Velocity of Shortening Relation? . . . . 117

5.4.4 Heat Production During Forcible Stretching a Contracting Muscle. . . . . . . . . . . . . . . . . . 119

$5.4 .5 \quad$ Relaxation Heat. . . . . . . . . . . . . . . . . . . 120 


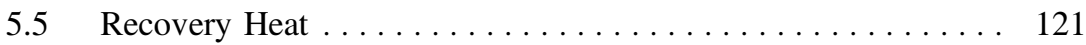

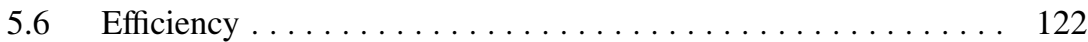

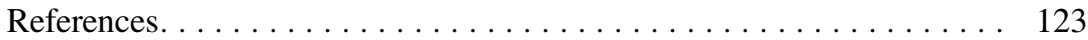

\section{Part II Locomotion: Motor-Machine Interaction}

6 External, Internal and Total Mechanical Work Done

During Locomotion . . . . . . . . . . . . . . . . . . . . . . . . . . . . . 129

$6.1 \quad$ External Work .......................... 129

6.1.1 Mechanical Energy Changes of the Center of Mass During Locomotion ................ 131

6.1.2 Assumptions Made in Calculating External Work from the Force Exerted on the Ground ........... 134

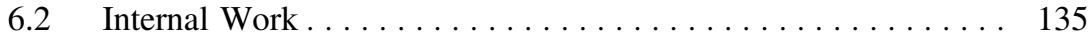

6.3 Total Work........................... 137

References. . . . . . . . . . . . . . . . . . . . . 138

$7 \quad$ Walking . . . . . . . . . . . . . . . . . . . . . . . 139

7.1 The Pendular Mechanism of Walking: A Way to Reduce External Work ............................ 139

7.2 Assessment of the Exchange Between Potential and Kinetic Energy....................... 140

7.3 Phase Shift Between Kinetic and Potential Energy .......... 146

7.4 Within the Step Pendular Energy Transduction in Human Walking . . . . . . . . . . . . . . . . . . . . . . 147

7.5 The Mechanism of Walking During Growth. . . . . . . . 148

7.6 Optimal and Freely Chosen Walking Speed ............ 150

7.7 The Mechanism of Walking in Different Animal Species...... 151

7.8 Effect of Step Frequency on the Mechanical Power Output in Human Walking. ....................... 151

7.9 Role of Gravity in Human Walking . . . . . . . . . . . . 156

7.10 Mechanics of Competition Walking .............. 158

7.11 Ergometric Evaluation of Pathological Gait . . . . . . . . . 161

References. . . . . . . . . . . . . . . . . . . . . . . . . . . . . 164

8 Bouncing Gaits: Running, Trotting and Hopping . . . . . . . 167

8.1 Transition from the Mechanism of Walking to the Mechanism

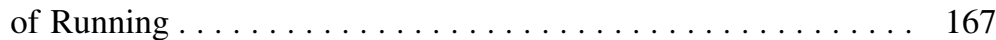

8.2 The Bouncing Mechanism of Progression. . . . . . . . . . . 168

8.3 Vertical Oscillation of the Center of Mass . . . . . . . . . . . 173

8.4 Physical Division of the Vertical Oscillation of the Center of Mass....................... 175

8.5 The On-Off-Ground Symmetry and Asymmetry of the Rebound.......................... 177 
8.6 The Landing-Takeoff Symmetry and Asymmetry of the Rebound. . . . . . . . . . . . . . . . . . . . . . . . 180

8.6.1 Height of the Center of Mass at Touch Down

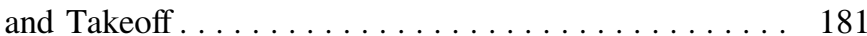

8.6.2 The Four Phases of the Bounce and the Transduction of Mechanical Energy During the Running Step . . . . . 182

8.6.3 Positive and Negative Work Durations . . . . . . . . . . 185

8.7 Landing-Takeoff Asymmetry of the Bouncing Step:

Asymmetric Motor or Asymmetric Machine? . . . . . . . . . . . 186

8.7.1 Different Machines with the Same Motor . . . . . . . . . . 187

8.7.2 Running Backwards: Soft Landing-Hard Takeoff . . . . 191

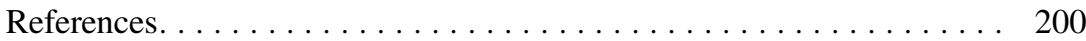

9 Effect of Speed, Step Frequency and Age on the Bouncing Step . . . . . . . . . . . . . . . . . . . . 203

9.1 Effect of the Running Speed on the On-Off-Ground Asymmetry and the Landing-Takeoff Asymmetry. . . . . . . . . . . 203

9.2 Effect of the Step Frequency on the Mechanical Power Output in Human Running . . . . . . . . . . . . . . . . . . . . 206

9.3 The Resonant Step Frequency in Human Running . . . . . . . . . 210

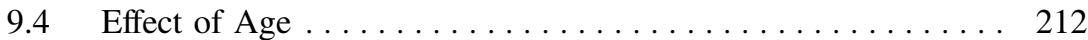

9.4.1 On-Off-Ground Symmetry and Asymmetry . . . . . . . . 212

9.4.2 Landing-Takeoff Asymmetry During Growth . . . . . . . 212

9.4.3 Landing-Takeoff Asymmetry in Old Age . . . . . . . . 216

References. . . . . . . . . . . . . . . . . . . . . . . . . . . . . . 220

10 Work, Efficiency and Elastic Recovery . . . . . . . . . . . . . 223

10.1 Mechanical Work Done by Adult Humans as a Function

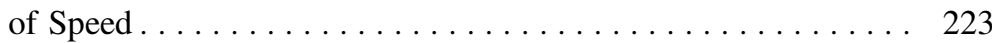

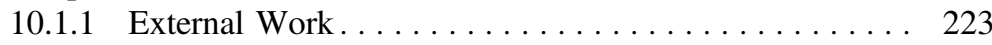

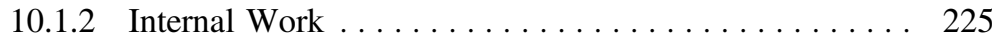

10.1.3 Total Work and Efficiency. . . . . . . . . . . . . . . 227

10.2 Running Children . . . . . . . . . . . . . . . . . . 230

10.2.1 External Work . . . . . . . . . . . . . . . . . . 230

10.2.2 Internal Work, Total Work and Efficiency.......... 237

10.3 Old Men Running. . . . . . . . . . . . . . . . . . . . . . . . 239

10.4 Effect of Body Mass on Elastic Recovery. . . . . . . . . . . . . 244

10.5 Effect of Gravity in Human Running . . . . . . . . . . . . . . 253

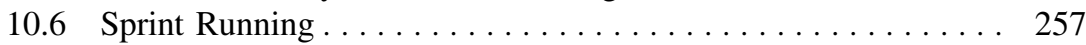

10.7 Vertical Jump . . . . . . . . . . . . . . . . . . . . . . . 264

10.7.1 Effect of Stretch Amplitude. . . . . . . . . . . . . . . . . 266

10.7.2 Jumping at Different Simulated Gravities . . . . . . . . . 268

10.7.3 Metabolic Energy Expenditure . . . . . . . . . . 271

References. . . . . . . . . . . . . . . . . . . . 271 